\title{
骨㿉合剂配合腰背肌功能锻炼治疗中 青年腰椎间盘突出症的临床观察
}

Bone Arthralgia Mixture Combined with Functional Exercise of Lumbar and Dorsal Muscles in the Treatment of Young and Middle-aged People

\author{
尹本敬 向俊宜 \\ Benjing Yin Junyi Xiang
}

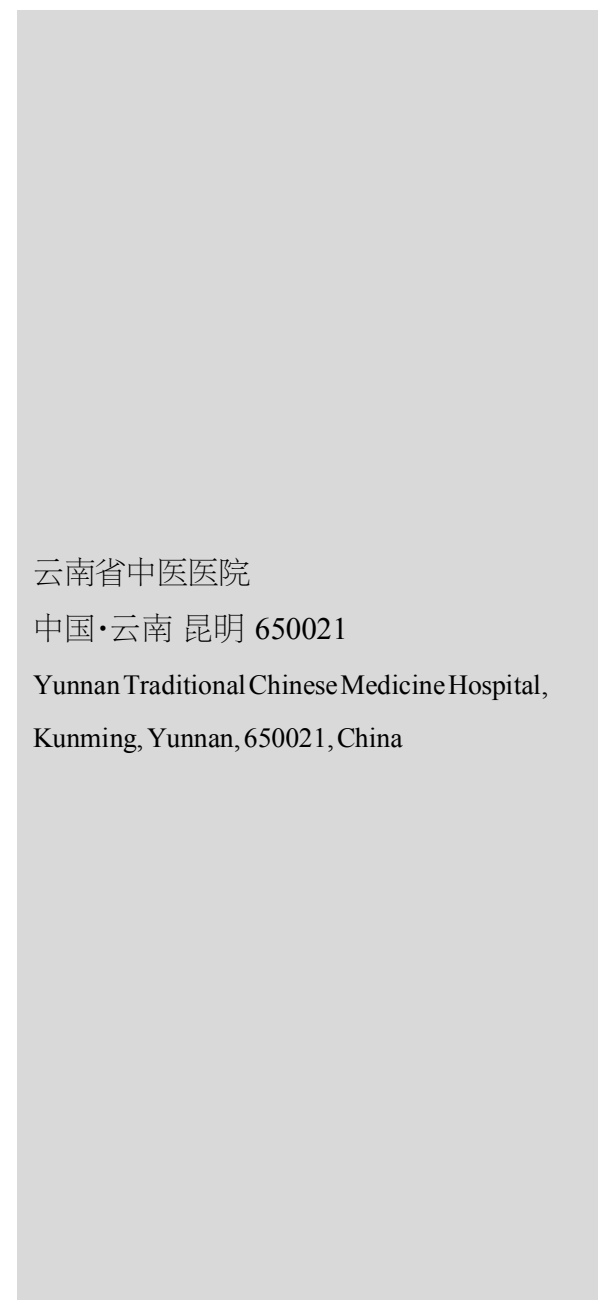

\section{1 引言}

腰椎间盘突出症 (Lumber Intervertebral Disc Herniation, $\mathrm{LDH}$ )是临床常见病、多发病, 主要是由于腰椎椎间盘退变变 性、纤维环破裂、髓核突出压迫刺激神经根和马尾神经导致以 腰痛、坐骨神经痛为主要临床表现的综合征, 好发于 20 50 岁, 男女约为 4 6: $1^{[1]}$ 。本病具有病程长、反复发作的特点, 严重 影响工作和生活质量, 本病多以非手术治疗为主。非手术治疗 包括药物治疗、针众治疗、推拿、牵引、神经阻滞治疗、物理疗
【摘要】为了探讨骨痹合剂配合腰背肌功能锻炼在中青年腰椎间盘突出症治疗中的远期 疗效和腰背肌功能铅炼在延缓腰椎间盘突出症复发的意义,选择经腰椎 MRI 确诊的 60 例 腰椎间盘突出症患者,随机分为治疗组和对照组。结果显示,骨痹合剂配合腰背肌功能锻炼 治疗, 对 VAS 以及腰椎 JOA 评分系统的影响均有明显统计学差异; 治疗组与对照组在治疗 后 7 天、14 天疗效无明显差别 $(P>0.05)$; 出院 1 年后, 治疗组疗效明显高于对照组 $(P<0.05)$, 随访 1 年, 治疗组复发率均低于对照组。由此得出, 骨痹合剂配合腰背肌功能锻炼在中青年 腰椎间盘突出症治疗中有很好的远期疗效。

【Abstract】In order to explore the long-term effect of bone arthralgia mixture combined with functional exercise of lumbar and dorsal muscles in the treatment of lumbar disc herniation in young and middle-aged people and the significance of functional exercise of lumbar and dorsal muscles in delaying the recurrence of lumbar disc herniation, 60 patients with lumbar disc herniation diagnosed by lumbar MRI are randomly divided into treatment group and control group. The results show that the effect of Gubi Mixture combined with functional exercise of lumbar and dorsal muscles on VAS and JOA scoring system of lumbar spine is significantly different; there is no significant difference between the treatment group and the control group in the efficacy of 7 days and 14 days after treatment $(P$ $>0.05$ ); one year after discharge, the curative effect of the treatment group is significantly higher than that of the control group $(P<0.05)$, followed up for 1 year, the treatment group. The recurrence rate is lower than that of the control group. It is concluded that bone arthralgia mixture combined with functional exercise of lumbarand dorsal muscles has a good long-term effect in the treatment of lumbar intervertebral disc herniation in young and middle-aged people.

【关键词】腰椎间盘突出症; 腰背肌功能锻炼; 骨痹合剂; 远期疗效

【Keywords】 lumbar intervertebral disc herniation; functional exercise of lumbar and dorsal muscles;bone arthralgia mixture;long-term efficacy

【DOI】10.36012/pmr.v1i1.146

法、运动疗法等 ${ }^{[2]}$ 。腰背肌功能锻炼属于运动疗法。 坚持腰背肌 的锻炼不仅可以巩固腰椎间盘突出症的治疗效果，还可以防 止或延缓复发 ${ }^{[3]}$ 。笔者自 2017 年 1 月一 2018 年 1 月采用骨痹 合剂配合腰背肌功能锻炼治疗腰椎间盘突出症 30 例, 疗效满 意,现报道如下。

\section{2 临床资料}

\section{1 病例资料}

选择 2017 年 1 月一 2018 年 1 月 60 例经腰椎 MRI 检查 
医学理论研究 Medical Theory Research

确诊为腰椎间盘突出症患者 60 例, 年龄 20 55 岁, 男 34 例, 女 26 例, 病程 1 个月至 3 年, 其中, L4-5 椎间盘突出 28 例, L5-S1 椎盘盘突出 32 例, 按病人就诊及入院的顺序分为 2 组, 治疗组 30 例、对照组 30 例, 组在性别、年龄、病程、突出节段 等方面, 无显著性差异 $(P>0.05)$, 具有可比性。

\section{2 诊断标准 ${ }^{[4]}$}

(1)病史: 有慢性劳损史、外伤史和腰痛史; (2)症状及体征: 腰部疼痛酸软, 下肢麻木同时存在坐骨神经区疼痛, 腰椎侧 凸, 腰部活动明显受限, 腰骮部及棘突间压痛、叮击痛, 直腿抬 高试验及加强试验阳性, 股神经牵拉试验阳性; (3)辅助检查: 腰椎 X 平片、CT、MRI 确诊腰椎间盘突出。

\section{3 方法}

\section{1 治疗方法}

选取符合条件的 60 例中青年腰椎间盘突出症患者, 依据 随机数字表法分为治疗组和对照组, 2 组均采用口服骨痹合 剂。其中, 治疗组 30 例配合腰背肌功能锻炼, 骨痹合剂是院内 制剂批准文号: 滇药制字(Z)04A01887,100mL瓶。治疗第一天 开始口服, 2 次 $\mathrm{d}$, 连续服用 4 周。对照组单纯口服骨痹合剂, 用 法用量及服药时间同治疗组。腰背肌锻炼: 第一步,五点支撑半 桥训练: 患者去枕仰卧位, 屈膝屈髋, 双肩肘和双足跟支撑, 抬 起臀部维持。第二步, 小飞燕训练:患者俯卧,抬头挺胸, 双上肢 后伸, 双下肢后伸抬起, 仅使腹部着床, 身体呈弓形, 如飞燕点 水。以上 2 法训练次数采用 TENS 原则, 即每组做 10 次, 每次 坚持 5 10 秒, 次间休息 10 秒。第三步,患者仰卧位, 双下肢伸 直, 做直腿抬高练习 10 20 遍, 以上锻炼要求早晚各 1 次。

\section{2 疗效评定方法}

所有患者在治疗前后, 行目测类比评分法 $(\mathrm{VAS})^{[5]}$ 和腰椎 JOA 评分系统配平估患者改善状况。VAS 评分标准: 10 分, 为无 痛; (2)1 3 分为有轻微的疼痛, 患者能忍受; (3) 4 6 分为疼痛并 影响睡眠, 尚能忍受; (4)7 10 分为有较强烈的疼痛, 疼痛难忍。

\section{3 结果处理}

统计学处理采用 SPS 22.0 统计分析处理数据, 所有计量 资料以等级资料的 2 样本比较采用 $t$ 检验。

\section{4 结果}

治疗组和对照组在治疗前后 VAS 评分比较, 差异有统计 学意义 $(P<0.05)$, 治疗组在疼痛改善上优于对照组。腰椎 JOA 评分 7 天、 14 天、一年疗效结果如下:治疗后 7 天治疗组优良 率为 $73.3 \%$, 对照组为 $63.3 \%$; 治疗后 14 天治疗组优良率为
$80.0 \%$, 对照组为 $73.3 \%$; 1 年治疗组优良率为 $76.6 \%$, 对照组 为 $43.3 \%$ 。从以上统计结果可以看出, 治疗后第 7 天与第 14 天 2 组疗效对比差异无统计学意义 $(P>0.05)$; 患者出院后 1 年, 2 组评分差异有统计学意义 $(P<0.05)$ 。

\section{5 讨论}

腰椎间盘突出症主要病理改变是椎间高度减少或丧失, 椎间隙变窄, 椎间盘退变, 纤维环破裂髓核突出, 刺激压迫神 经根出现腰痛及坐骨神经症状。中医理论认为, 腰椎间盘突出 症属于 “腰腿痛” “痹症” 的范畴[], 其发病的主要原因是外感六 淫之邪, 劳损、跌扑损伤治气滞血瘀, 经脉闭塞不通或肝肾亏 虚经脉失养。骨痹合剂是我院骨伤科经长期临床实践以及结 合现代医学研究治疗腰腿痛而研制成的院内制剂, 疗效确切。 方中主要由独活、㒸活、桑寄生、杜仲、续断、丹参、红花、细辛、 甘草等 15 味中药材配制而成, 方中独活祛风湿, 止痹痛; 桑寄 生补肝肾, 强筋骨为君药; 杜仲补益肝肾, 强笳壮骨; 续断行血 脉, 续筋骨; 丹参、红花活血祛瘀止痛; 㒸活祛风胜湿、细辛祛 风散寒, 温经止痛等药物共为佐药, 甘草调和诸药, 为使药。骨 痹合剂对于患者疼痛缓解、改善功能缓解腰腿疼痛有明显的 作用 ${ }^{[8]}$ 。腰背肌锻炼及直腿抬高运动, 不仅能增强腰背肌肌力, 改善腰背韧性和调整肌肉的张力, 还可以加强脊柱关节的稳 定性, 改善神经水肿刺激症状。坚持腰背肌的锻炼不仅可以巩 固腰椎间盘突出症的治疗效果, 还可以防止或延缓复发, 在临 床上有一定的推广意义。

\section{参考文献}

[1]吴在德,吴肇汉.外科学[M].北京: 人民卫生出版社,2010.

[2]王芝琴, 李友寒, 张涛. 以运动疗法为主综合治疗腰椎间盘突出 症 20 例[J].南华大学学报,2007,35(5):778-779.

[3] 黄建洪.腰背肌功能训练在预防腰椎间盘突出症复发中的作用 [J].中国康复,2003,18(3):198.

[4]胡有谷.腰椎间盘突出症[M]. 北京: 人民卫生出版社,2011.

[5]Fukui M, chiba K, Kawakami M,et a1. Japanese Orthopaedic Associa-tion Cervical Myelopathy Evaluation Questionnaire[J].J Orthop Sci, 2008,13(1):25-31.

[6]Yonenobu K, Okada K, Fuji T, et a1. Cause of Neuro-logic Deterioration Following Surgical Treatment of Cervicd Myelopathy [J].Spine, 1986(11):818-823.

[7]莫文,李晓锋,叶洁,等.施杞论治腰椎间盘突出症的经验[J].上海 中医药杂志,2017,51(10):1-4.

[8]赵文韬,包可,王琦.骨痹合剂治疗膝骨关节炎疗效观察 [J].现代 中西医结合杂志,2009(13):1492-1493. 